\title{
Active Neutron Monitoring of Nuclear Fuel Cycle Fissile Materials
}

\author{
V.F. Batyaev, M.D. Karetnikov, S.V. Sklyarov
}

Dukhov Research Institute of Automatics (VNIIA), 127055 Moscow, Russia

\begin{abstract}
A decommissioning of nuclear fuel cycle facilities is inseparable from the problems of radioactive waste disposal. One of these problems is the categorization of a waste according to the content of beta- and alpha-emitters. Beta-emitters can be identified by existing technologies; however, the trouble arises when detecting alpha-emitting elements, primarily the long-lived members of the actinium chain with the specific activity of $\mathrm{kBq} / \mathrm{kg}$ when they are spread inside a structural material. The report considers an application of an active neutron method-a differential die-away technology for reliable control of small quantities of FM.

The essence of this method consists in sounding the interrogated item by pulsed thermal neutrons and recording the induced fission neutrons. The ratio of the number of fission neutrons to the number of source neutrons gives the normalized number of fission neutrons that is linked to the FM mass in the interrogated object. The work presents the scheme and principle of operation of an experimental device, as well as the results of measurement of concrete structures that contain internal traces of fissile materials. Analysis of the results shows that the proposed method allows the detection of $\sim 6 \mathrm{mg}$ of fissile material per $\mathrm{kg}$ of concrete with possible localization (cartogram) of the contaminated area.
\end{abstract}

Keywords - Fissile materials, Neutron generator, radioactive wastes, neutron detector.

\section{INTRODUCTION}

Detection of small amounts of fissile materials (FM) is a key mission for determining the degree of contamination of decommissioned buildings and structures where FM were handled [1]. Since the cost of burial of low-level (LLW) and high-level (HLW) radioactive wastes differs by more than an order of magnitude, measuring the FM content in the structures of decommissioned buildings is a very urgent task. To classify such structures as LLW, the minimum content of alpha emitters (not transuranium) shall be as low as 100 $\mathrm{kBq} / \mathrm{kg}$, which corresponds to $\sim 30 \mathrm{mg}$ of ${ }^{235} \mathrm{U}$ per $\mathrm{kg}$ of concrete taking into account the full range of alpha emitters. It should be noted that the ${ }^{235} \mathrm{U}$ enrichment has insignificant influence on the total activity of alpha emitters in concrete.

In the existing practice, the activity measurement usually involves a spot sample from the LLW package, passing preliminary multi-stage preparation: grinding, dissolving, evaporation, averaging, etc. Sampling of a representative quantity is difficult for LLW in form of metal structures, construction debris, etc. The correctness of the results of measurement and their duration leaves much to be desired. There is a problem of temporary storage of waste batches, which is particularly urgent for enterprises with large volumes of LLW circulating at various stages of processing.

Passive methods of FM control in LLW based on detection of spontaneous fission products are much more rapid and do not require sampling. However, the gamma radiation of ${ }^{235} \mathrm{U}$ or ${ }^{239} \mathrm{Pu}$ has a weak penetrability and often a relatively low intensity for practical measurements. In addition, the spectral peaks of ${ }^{235} \mathrm{U}$ and ${ }^{239} \mathrm{Pu}$ interfere with the characteristic spectral lines of many radioactive isotopes in LLW (e.g., ${ }^{133} \mathrm{Ba},{ }^{137} \mathrm{Cs}$, $\left.{ }^{131} \mathrm{I}\right)$, which can completely mask gammaradiation from FM even when $\mathrm{HPGe}$ high-resolution spectrometers are used. ${ }^{235} \mathrm{U}$ and ${ }^{239} \mathrm{Pu}$ feature small neutron yield of spontaneous fission, therefore, unlike ${ }^{240} \mathrm{Pu}$ and other even isotopes, they cannot practically be detected by neutron radiation. Thus, when small amounts of FM are distributed in the volume of a concrete structure, it is very difficult or practically impossible to detect low content of ${ }^{235} \mathrm{U}$ or ${ }^{239} \mathrm{Pu}$ using passive control methods.

Active methods based on detecting the products of nuclear reactions in FM sounded by penetrating radiation can potentially provide reliable control of small quantities of fissile materials. More than dozens nuclear-physical methods have been proposed that are suitable to different extent for solving the missions [3]. The analysis shows that the best accuracy, rate and reliability of the results are provided by the photonuclear and the differential die-away technologies. Choice of the method depends, first of all, on the type of matrix (material) where FM are concealed. It is associated with the features of penetration of the sounding and response (useful) radiation. For a matrix with a small atomic number and a high content of hydrogen atoms, it is preferable to use the photonuclear technology [4]; for a matrix with a medium and large atomic number (iron, concrete, lead), the differential die-away technology provides the best sensitivity. Moreover, differential die-away technology is insensitive to the fertile $\left({ }^{238} \mathrm{U},{ }^{232} \mathrm{Th}\right)$ materials that is essential for our task. The essence of this method consists in sounding the interrogated item by pulsed thermal neutrons and recording the induced fission neutrons. The neutron detector registration threshold is $0.4 \mathrm{eV}$ due to the cadmium screening, thus the detectors responde to fission neutrons but insensitive to sounding 
thermal neutrons. The neutron moderator, measurement chamber and detector assembly are configured in such a way that the sounding neutrons and fission neutrons are timecorrelated and they can be separated by measuring their time behavior [2]. The counting rate of neutron detector can be represented as

$$
\Phi(t)=A \cdot \exp (-\alpha t)+B \cdot \exp (-\beta t)+\Phi_{0},(1)
$$

where $A, B$ are pre-exponential factors; $\alpha$ is the neutron decay constant determined by the moderator thickness behind the cadmium shield; $\beta$ is the decay constant of the thermolized neutrons of FM determined by the neutron properties of the set-up; $\Phi_{0}$ is the sum of the responses of delayed neutrons, spontaneous fission neutrons, neutrons from $(\alpha, n)$ reactions, as well as the background of the premises. The ratio of the number of fission neutrons to the number of source neutrons gives the normalized number of fission neutrons (normalized amplitude coefficient $B / A$ ), that does not depend on the neutron generator yield. The specified value of $B / A$ is linked to the FM mass placed in the interrogated object [2].

\section{EXPERIMENTAL SETUP OF FM DETECTOR IN CONCRETE}

To study the possibility of detecting small amounts of FM in a concrete matrix (a model of buried LLW) the experimental FM detector based on the differential die-away technology (Fig. 1) was designed using the ING-07T D-T neutron generator (produced by VNIIA, Russia) as a source of sounding neutrons. The generator provided a pulsed flux of 14-MeV neutrons with a $800 \mathrm{~Hz}$ pulse frequency and a $14 \mu \mathrm{s}$ neutron pulse width. An average intensity was as high as $5 \cdot 10^{8}$ $\mathrm{n} / \mathrm{s}$. Fission neutrons were detected by five boron gas-filled counters of thermal neutrons of the SNM-KCh type (manufactured by NIITFA, Russia) surrounded by a moderator and a cadmium filter. One boron counter without cadmium filter was used to monitor the neutron flux from the neutron generator. The choice of boron counters is due to their low sensitivity to gamma radiation, since measurements in the realistic environment can take place under a strong gamma background. The contaminated and pollution-free parts of concrete constructions were imitated by: a concrete $850 \times 530 \times 280 \mathrm{~mm}^{3}$ cube; $100 \times 100 \times 50 \mathrm{~mm}^{3}$ concrete samples (12 pieces without uranium; 12 pieces with a ${ }^{235} \mathrm{U}$ content uniformly distributed with an average concentration of 6 $\mathrm{mg} / \mathrm{kg}$ ); 42 pieces of $3.5 \mathrm{mg}$ point samples of ${ }^{235} \mathrm{U}$ (147 mg of 235

$\mathrm{U}$ in total)). The signals were detected using a MAVS-16 time analyzer (produced by VNIIA, Russia) which allowed detecting the number of counts in 256 consecutive time windows of arbitrary width (the window number is counted from the sync pulse from the neutron generator). In the experiments the width of each time window was chosen to be $4 \mu$ s for a complete analysis of the counting rate of counters as function of time.

Neutron counters were installed in a chamber that simultaneously served as a moderator for $14 \mathrm{MeV}$ source neutrons and a moderator for fission neutron counters. The configuration of the camera has been optimized to ensure maximum sensitivity of fission neutron detectors and a minimum background level.

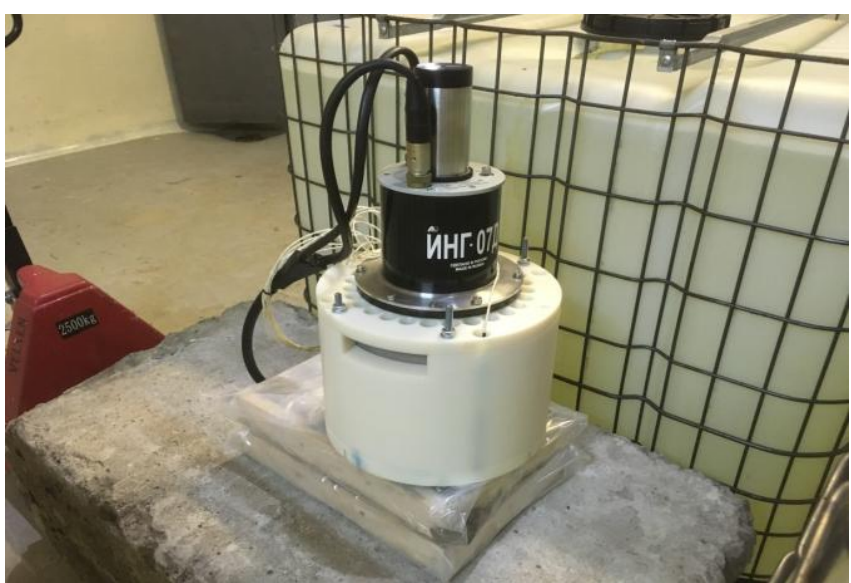

Fig.1 Experimental detector of FM in concrete structures.

An example of the experimental data processed by smoothing function is given in Fig. 2. The figure of merit $F O M=-$ was used as a criterion for the effect $F_{1}$ above

the background $F_{2}$. To maximize the effect-to-background ratio the optimal time interval $\Delta T=t_{2}-t_{1}=500 \mu \mathrm{s}$ for integrating the effect (number of counts in the presence of FM) and the background was applied.

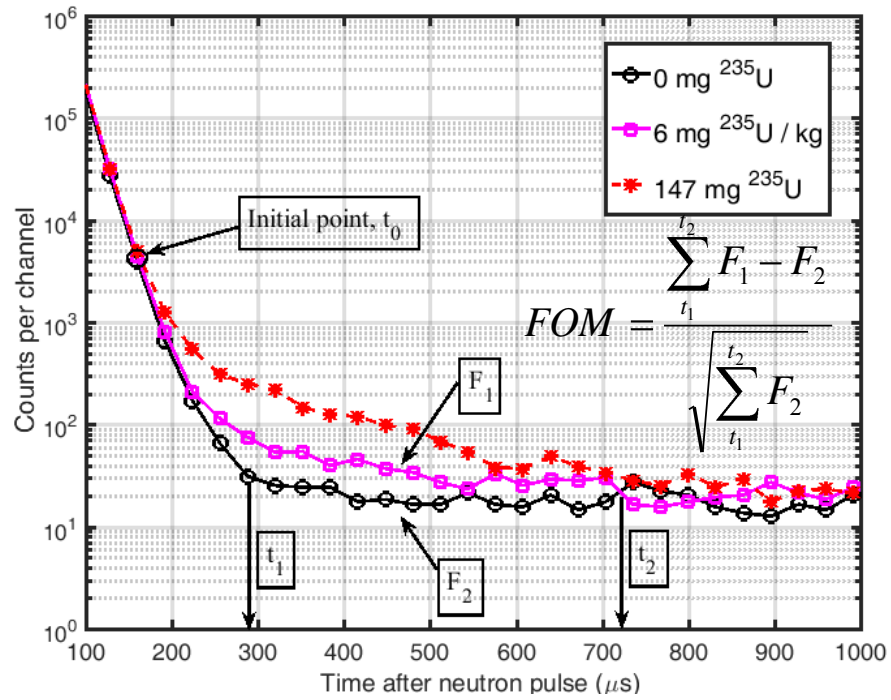

Fig. 2 Example of experimental number of counts per $16-\mu \mathrm{s}$ channel as function of measurement time.

The sensitivity of FM detector in case of a point distribution of ${ }^{235} \mathrm{U}$ on the concrete surface was close to $6 \mathrm{mg}$ for $\mathrm{FOM}=3$.

The FM detector allowed localization the FM, which is very important for the measurement of concreted LLW with nonhomogeneous density of FM. This is physically possible due to the geometric factor $\left(1 / R^{2}\right)$ and the attenuation of the diffuse neutron flux with a distance. Measurements with the stepwise shift of the FM detector from FM were carried out to demonstrate the possibility of determining the FM position coordinates in the concrete structure. FM was in a form of a long tape with target indicators applied on it (a total weight of ${ }^{235} \mathrm{U}$ of $147 \mathrm{mg}$ ), and was placed in a 6-cm-deep hole in concrete. The dependence of FOM on the shift of the FM detector is demonstrated in Fig. 3. It can be seen that $F O M=27$ 
when the FM detector is placed at $7 \mathrm{~cm}$ from FM. In this case, the minimum detectable mass of FM is $15 \mathrm{mg}$. The sensitivity drops by 3.2 times at a distance of about $30 \mathrm{~cm}$.

\section{NUMERICAL SIMULATION OF FM DETECTION}

To further substantiate the possibility of detecting the volumetric distribution of FM inside a concrete structure, a numerical simulation of FM detection with a geometry similar to the experiments performed was carried out. $147 \mathrm{mg}$ of ${ }^{235} \mathrm{U}$ was placed inside the $850 \times 530 \times 280 \mathrm{~mm}^{3}$ concrete block in a tunnel $400-\mathrm{mm}$ long at a $60-\mathrm{mm}$ distance from the surface. The neutron flux density was calculated in the cells of a computational grid with cell sizes of $20 \times 20 \times 20 \mathrm{~mm}^{3}$ to study the spatial distribution of neutrons from FM with energies above $1 \mathrm{eV}$. The choice of this neutron energy range is due to a relatively high probability of their detection by the boron counters located behind the cadmium shield. The neutron source with the Watt spectrum was set as an extended one, geometrically coincident in position and dimensions with FM. This made it possible to determine the neutron flux density of FM in the entire volume of the concrete (including the surface corresponding to the location of the FM detector) of the structure and compare the results of epithermal neutron flux attenuation with the experimental results of measuring the minimum detectable FM mass. Fig. 4 illustrates the results of simulation for one surface of the concrete block.

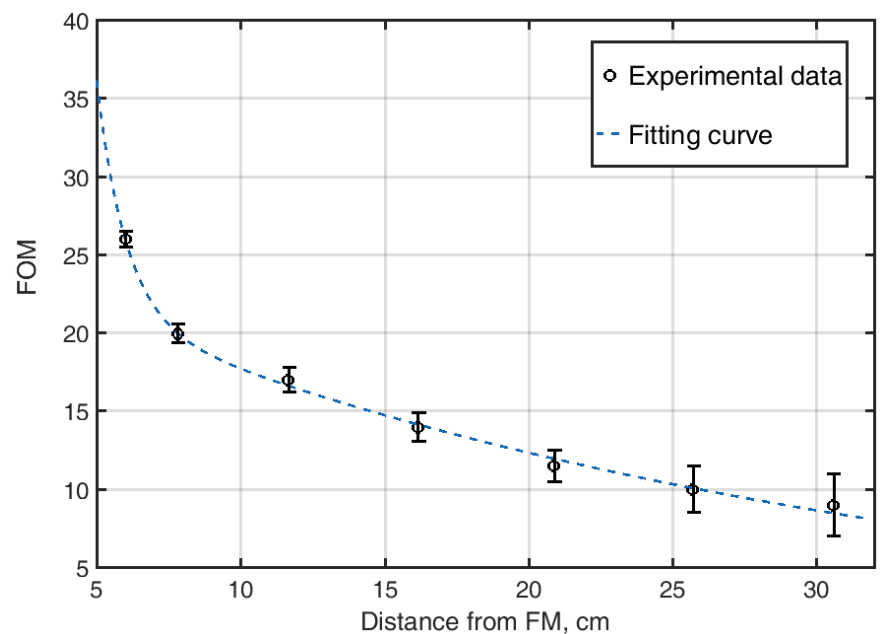

Fig. 3 The dependence of FOM on the shift of the FM detector.

The data obtained show that the attenuation of the epithermal neutrons flux along the $X$ axis along the surface of the concrete structure is $\sim 3.2$ times over a length of $30 \mathrm{~cm}$ from the center of the FM location. This result correlates well with the measured function of the FM detector sensitivity (Fig. 3) vs the distance to FM. Moreover, to improve the convergence of the experimental and calculated results, it is necessary to obtain the distribution of the neutron flux density not on the surface of the concrete structure, but after passing through the cadmium and polyethylene shield, thereby simulating the neutrons hitting the detector.

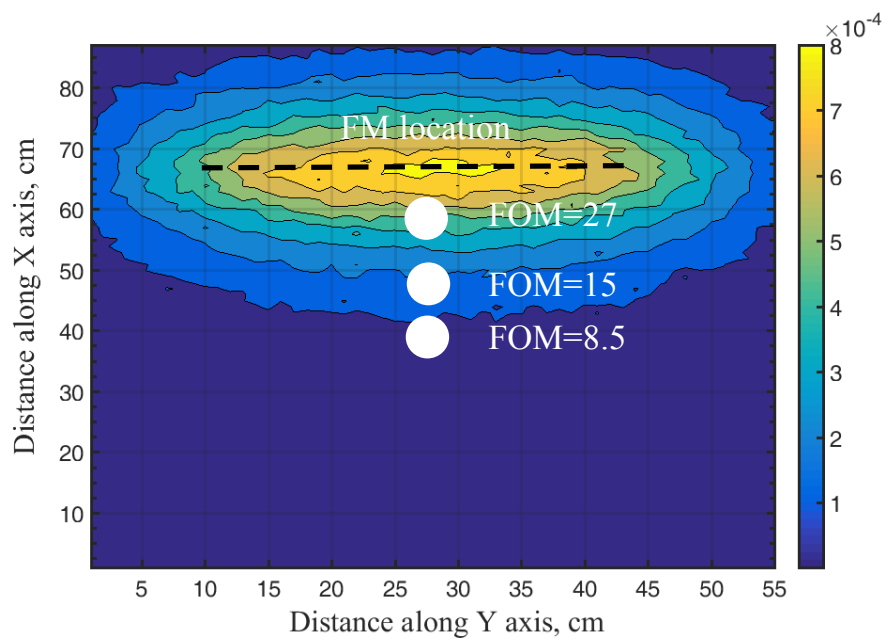

Fig. 4 Flux density of epithermal neutrons on surface of concrete structure per one source neutron. The value of FOM corresponds to the position of FM detector.

The algorithm for spatial detection of the FM position in concrete can be based on the representation of the experimentally obtained count rate of detector, $E_{i}$, as a product of the counters response matrix by a unit amount of uranium in the previously known cells and the vector of weight factors, $M_{U j}$, corresponding to the amount of FM in the cells.

Count rate of counter $\mathrm{i}$ :

$$
E_{i}=w_{i j} \cdot r_{j}=w_{i j} \bullet\left[\sigma_{U} \cdot \Phi_{j} \cdot M_{U j} \cdot \frac{N_{a}}{A}\right]=R_{i j} \bullet M_{U j}
$$

Where $j$ is number of cell in object; $w_{i j}$ is counter sensitivity matrix as a measure of counter $i$ response to neutrons from one uranium fission in cell $j ; r_{j}$ is fission rate of uranium in cell $\mathrm{j}$; $\sigma_{\mathrm{U}}$ is uranium fission cross-section; $\Phi_{j}$ is neutron flux density in cell $j$ during response measurement; $M_{U j}$ is uranium mass in cell $j ; N_{A}$ is Avogadro number; $A$ is uranium atomic mass; $R_{i j}$ is counter response (count rate) matrix to a unit of uranium in cells; • is matrix multiplication operation

This expression in the matrix form can be represented as follows:

$$
\left[\begin{array}{ccc}
R(1,1) & \ldots & R\left(1, j_{\max }\right) \\
\ldots & \ldots & \ldots \\
\ldots & \ldots & \ldots \\
\ldots & \ldots & \ldots \\
R\left(i_{\max }, 1\right) & \ldots & R\left(i_{\max }, j_{\max }\right)
\end{array}\right] \times\left[\begin{array}{c}
M(1) \\
\ldots \\
M\left(j_{\max }\right)
\end{array}\right]=\left[\begin{array}{c}
E(1) \\
\ldots \\
\ldots \\
\ldots \\
E\left(i_{\max }\right)
\end{array}\right]
$$

\section{CONCLUSION}

Experiments have shown that the minimum detectable mass of ${ }^{235} \mathrm{U}$ by five counters is $6 \mathrm{mg} / \mathrm{kg}$ with a uniform distribution over the concrete samples. In the future, our intension is to increase the number of counters up to 64 to improve the sensitivity of the setup and to study its applicability for measuring the amount and position of FM in various matrices. In addition, the paper presents an analytical method for determining the FM distribution in a concrete structure, which 
shall be further verified using a cellular concrete structure and a set of samples with different FM content.

The authors acknowledge participation of S.N. Florya, D.I. Vlasov (FSUE "RosRAO"), V.F.Danilov and G.V.Poletov (NIITFA industrial JSC) regarding their help in preparing and arranging demonstration experiments under process conditions and valuable discussions.

\section{REFERENCES}

[1] Radioactive waste.-[electronic resource.] URL: www.norao.ru/waste/ (accessed 20.02.2019).

[2] Viacheslav F.Batyaev, Sergey S. Sklyarov., Comparative Analysis of Nondestructive Methods to Control Fissile Materials in Large-size Containers. International Conference Nuclear Data 2016, Bruges, Belgium, 2016

[3] Gozani T. Non Destructive Assay of Spent Fuel for Determination of Residual Fissile Content // Journal of Instruments for Nuclear Materials Management, V. 5, 1976, P. 514-524.

[4] Gmar M., Jeanneau F., Laine F. et al. Assessment of Actinide Mass Embedded in Large Concrete Waste Packages by Photon Interrogation and Photofission // Applied Radiation and Isotopes, V. 63, 2005, P. 613619. 